\title{
Cómo Integrar la Ciencia de los Materiales en los Tres Niveles Educativos a través de la Preparación y Observación de Cristales Macroscópicos
}

\author{
Oriana D'Alessandro, María V. Curia y Laura E. Briand \\ Centro de Investigación y Desarrollo en Ciencias Aplicadas-Dr. J. J. Ronco - CONICET - Facultad \\ de Ciencias Exactas - Universidad Nacional de La Plata, Centro Científico Tecnológico La Plata- \\ CONICET, Calle 47 N $^{\circ}$ 257, B1900AJK La Plata, Buenos Aires-Argentina
}

\begin{abstract}
Resumen
El presente trabajo analiza cómo integrar la ciencia de los materiales en los tres niveles educativos, desde el nivel primario hasta el universitario, usando un experimento clásico. Se desarrolla la metodología de síntesis de cristales de sulfato de cobre a partir del crecimiento lento de los gérmenes de cristalización inmersos en una solución saturada de dicha sustancia. A partir de este experimento se discuten e integran los conceptos de solución, solubilidad, celda unidad, tipos de cristales y estructura de la materia. Adicionalmente, se discute el nivel de aprendizaje de la química que es posible transmitir a los alumnos de los distintos niveles educativos y se relaciona con la capacidad cognitiva de los estudiantes
\end{abstract}

Palabras clave: educación, ciencia, materiales, cristales, hábito cristalino

\section{How to Integrate the Fundamentals of Materials Science at the Three Educational Levels through the Synthesis and Observation of Macroscopic Crystals}

\begin{abstract}
This work analyzes the idea of integrating the fundamentals of materials science at the three educational levels, from elementary school to university level, using a classical experiment. A methodology for the synthesis and growing of hydrated cupper sulfate crystals has been developed. With this experiment various basic concepts such as solution, solubility, unit cells, types of crystals and structure of matter are discussed. Additionally, the level of chemistry that it is possible to transmit to the students in the different stages of their education is analyzed and related to the cognitive capacity of the students.
\end{abstract}

Keywords: education, science, materials, crystals, crystal habit 


\section{INTRODUCCIÓN}

Es evidente que existe una profunda crisis en la enseñanza de la ciencia, la que se detecta por opiniones desfavorables de adultos que la recuerdan como incomprensible y de estudiantes que se refieren a ella del mismo modo. La crisis también se pone en evidencia por la disminución del número de inscriptos en las carreras universitarias relacionadas con las ciencias exactas (Izquierdo Aymerich, 2004).

Las ciencias surgen de la actividad humana desde sus inicios hasta la actualidad, de la formulación de preguntas y de la búsqueda de respuestas. Pero el estudiante, no puede plantear preguntas y aprender ciencias sin actividad experimental en el aula y los profesores son los que deben promover estas actividades.

En el aula, la enseñanza de la ciencia debe garantizar una dinámica que permita el conocimiento y comprensión de los fundamentos teóricos, la aplicación experimental de los mismos, la observación y análisis del fenómeno, la síntesis y evaluación de los resultados y por último, la adecuada expresión escrita de las conclusiones (Bailey, 2008).

En este contexto, la química es una ciencia netamente empírica por lo cual, su enseñanza requiere un trabajo de laboratorio integrado es decir, que unifique varias técnicas, procedimientos y conceptos organizados lógicamente. Por ejemplo, un mismo experimento puede involucrar una metodología de síntesis, varios métodos de purificación y caracterización del producto, la determinación de propiedades físicas y hasta el estudio cinético y termodinámico del fenómeno. De hecho, la experimentación integrada enfatiza la idea que las diferentes técnicas no pertenecen a un área específica de la química sino que pueden aplicarse al estudio y resolución de problemas en cualquier área de la ciencia (D'Alessandro et al., 2008).

En esta línea pedagógica, el presente trabajo propone el cultivo de cristales de sulfato de cobre, y la transmisión de los conocimientos relacionados a los tres niveles educativos (primario, secundario y universitario) a través de la integración teórico-experimental.

\section{FUNDAMENTOS TEÓRICOS}

En principio, el cultivo de cristales requiere del conocimiento de los conceptos de solubilidad y estructura cristalina, los cuales serán brevemente comentados a continuación.

La solubilidad es la máxima cantidad (expresada en gramos) de un soluto que puede disolverse en una cantidad dada de solvente, a una temperatura determinada y se expresa como gramos de soluto por cada $100 \mathrm{ml}$ de solvente. Se pueden considerar tres tipos de soluciones dependiendo de la cantidad de soluto presente en ellas: solución diluida; saturada y sobresaturada (estado de no-equilibrio). Las soluciones diluidas contienen menos soluto que el que es posible disolver en el solvente a una determinada temperatura. Al aumentar gradualmente la cantidad de soluto en la solución se alcanza un estado de equilibrio conocido como "solución saturada" en el cual, no es posible disolver más soluto. Los sistemas que contienen mayor cantidad de soluto que la correspondiente a la solución saturada son inestables y por lo tanto, todo el exceso de soluto disuelto precipita transformándose en un sistema heterogéneo formado por una solución saturada en contacto con cristales de soluto. Así se forman una gran cantidad de cristales microscópicos que crecen rápidamente de manera imperfecta. Para formar cristales grandes y más perfectos, el solvente de la solución se debe dejar evaporar lentamente como se mostrará en la próxima sección (Brown et al., 2004).

La forma externa regular de los cristales sugiere una estructura interna ordenada. De hecho, la repetición periódica por la que se describe la estructura interna de los cristales viene representada por un conjunto de traslaciones en las tres direcciones del espacio, de tal forma que el cristal puede considerarse como un apilamiento, en tres dimensiones, de bloques idénticos. Cada bloque, de una forma y tamaño determinados, se denomina celda unidad (Chang, 1999; Brown et al., 2004). 
En dos dimensiones, una celda unidad es un paralelogramo con lados de longitud $a$ y $b$, que forman un ángulo $\gamma$ entre sí. En tres dimensiones, la celda unidad es un paralelepípedo con aristas de longitud $a, b$ y $c$ y ángulos $\alpha, \beta, \gamma$ entre las mismas. En este sentido, existen siete sistemas cristalinos basados en las relaciones de longitudes de las aristas $a, b, c$ y valores de los ángulos $\alpha, \beta, \gamma$. Particularmente, el sulfato de cobre cristaliza en el sistema triclínico cuya celda unidad posee las tres aristas de distinta longitud $(a \neq b \neq c)$ y ángulos también diferentes entre sí ( $\alpha \neq \beta \neq$ $\gamma)$.

\section{METODOLOGÍA PARA EL CULTIVO DE CRISTALES}

En esta sección se describen las etapas a seguir para cultivar cristales de sulfato de cobre pentahidratado a través de la evaporación lenta de una solución saturada (Holden y Morrison, 2001).

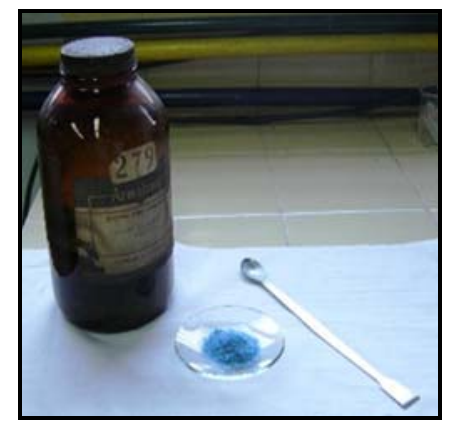

1- Pesar cuidadosamente

20,3 gr del reactivo en balanza granataria y disolver en $100 \mathrm{ml}$ de agua destilada tibia.

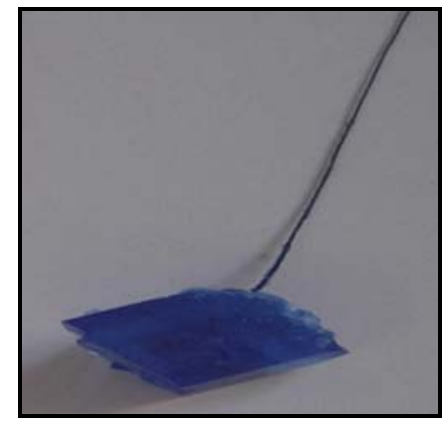

4- Enlazar cuidadosamente con un hilo cada uno de los pequeños cristales seleccionados.

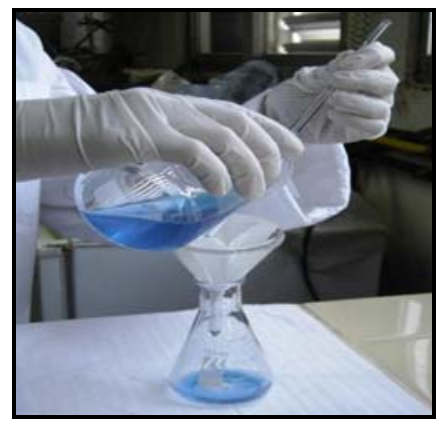

2- Filtrar en caliente, trasvasar el filtrado a un cristalizador y dejar enfriar lentamente. Se observa la formación de los pequeños cristales

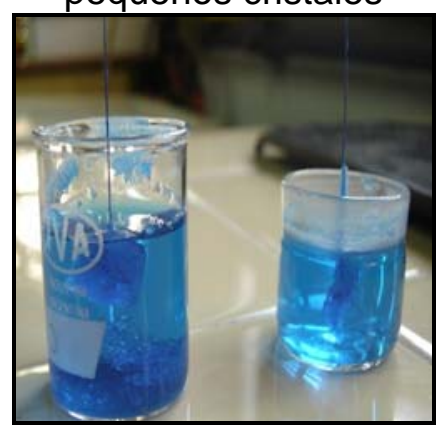

5- Suspender el cristal enlazado dentro de un recipiente adecuado y cubrirlo con la solución guardada.

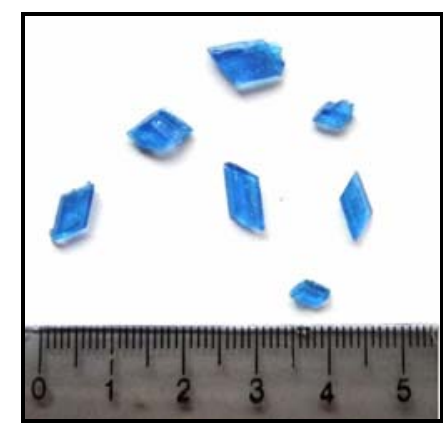

3- Seleccionar cuidadosamente los cristales más perfectos para ser utilizados como gérmenes de cristalización. No desechar la solución.

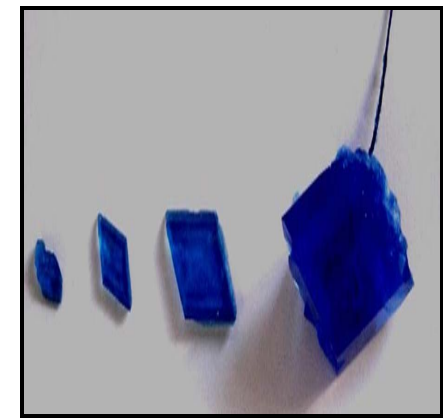

6- Observar el crecimiento de los cristales y comparar el tamaño final con el inicial. Observar con una lupa.

Esta metodología también se aplica al cultivo de cristales macroscópicos de cloruro de sodio (sistema cúbico), ferricianuro de potasio $(\mathrm{CN})_{6} \mathrm{FeK}_{3}$ (sistema monoclínico), nitrato de sodio $\mathrm{NaNO}_{3}$ (sistema hexagonal) entre otros.

\section{RESULTADOS Y DISCUSIÓN}

Según se comentó anteriormente, cada sustancia tiene una única forma cristalina correspondiente a un determinado ordenamiento espacial de los átomos que la componen. Sin embargo, algunos factores relacionados con las condiciones en las que tiene lugar el crecimiento del cristal conducen a la modificación del tamaño (crecimiento preferencial) de alguna de las caras del cristal aunque los ángulos entre caras adyacentes permanecen constantes. Este fenómeno fue descubierto por Nicolaus Steno quien formuló la ley de la constancia de los ángulos diedros (Greenwood, 1970). En la Tabla 1 se ejemplifica dicha ley partir de la determinación de la longitud de las aristas y el ángulo entre ellas de diversos cristales de sulfato de cobre obtenidos de acuerdo al procedimiento descripto en la sección anterior. 
Tabla 1: Longitud de las aristas y ángulos de diversos cristales de sulfato de cobre $\mathrm{CuSO}_{4} \cdot 5 \mathrm{H}_{2} \mathrm{O}$.

\begin{tabular}{lcccc}
\hline & \multicolumn{2}{l}{ Longitud de las aristas $(\mathrm{mm})$} & & $\begin{array}{c}\text { Ángulo entre } \\
\text { aristas } \boldsymbol{a} \text { y } \boldsymbol{b}\end{array}$ \\
\hline Cristal I & arista $\boldsymbol{a}$ & arista $\boldsymbol{b}$ & arista $\boldsymbol{c}$ & $\boldsymbol{\alpha}$ \\
\hline Cristal II & 6,02 & 4,60 & 1,60 & 59 \\
\hline Cristal III & 8,45 & 7,05 & 1,48 & 59 \\
\hline
\end{tabular}

\section{CONCLUSIONES: INTEGRACIÓN TEÓRICO-EXPERIMENTAL}

Sin duda, el cultivo de cristales en el laboratorio es el ejemplo perfecto para transmitir al estudiante los tres niveles de aprendizaje de la química que son: el nivel simbólico, submicroscópico o molecular y macroscópico (Mahaffy, 2004). En este contexto, el docente puede integrar los conceptos básicos de la estructura de la materia (átomo y molécula) y la construcción atómica progresiva de los sistemas materiales a través de la observación de la sobresaturación de la solución, la nucleación y el crecimiento de los cristales. Concretamente, el alumno podrá observar el tamaño de cristales en función del tiempo que ha estado en contacto con la solución saturada y concluir acerca del crecimiento progresivo del cristal tal como se observa en la última etapa de la metodología descripta en la sección anterior.

En el caso de los alumnos de la escuela primaria, la aplicación de los tres niveles de aprendizaje a través del presente trabajo está orientada fundamentalmente a la observación de los cristales para compararlos con sistemas materiales orgánicos. Sin embargo, los alumnos del nivel educativo secundario y universitario tienen herramientas para que el docente realice una discusión integradora de las observaciones experimentales y los diversos conceptos teóricos nombrados anteriormente a los fines de conectarlos, relacionarlos y extenderlos al cultivo de otros cristales.

\section{REFERENCIAS}

Bailey, P.D.; Should "Teacher Centred Teaching" Replace "Student Centered Learning"?, Chem. Educ. Res. Pract.: 9, 70-74 (2008).

Brown, T.L., H.E. LeMay, B.E. Bursten y J.R. Burdge, "Química La Ciencia Central", Pearson educación, 9na. Edición, USA, 430-434 y 491 (2004).

Chang, R.; "Química”, McGraw-Hill ed., 6ta. Edición, Mexico, 428-441 (1999)

D’Alessandro, O., M.V. Curia y L.E. Briand; Ciencia de los Materiales en los Niveles Educativos E.P.B: y E.S.B. Preparación y Observación de Cristales Macroscópicos, Actas de las VII Jornadas de Enseñanza Universitaria de la Química y XIV Reunión de Educadores en la Química, 20 al 23 de mayo de 2008, Olavarría (Buenos Aires, Argentina), publicado en CD con el código SII-3. (2008).

Greenwood, N.N.; Cristales iónicos, defectos reticulares y no estequiometría, ed. Alambra, 1ra edición, Madrid (1970).

Holden, A. y P. Morrison; "Crystals and Crystal Growing", The MIT Press, Cambridge, Massachusetts, 13ra Edición, USA (2001).

Izquierdo Aymerich, M.; Un Nuevo Enfoque de la Enseñanza de la Química: Contextualizar y Modelizar. J. Arg. Chem. Soc.: 92 (4/6), 115-136 (2004).

Mahaffy, P.; The Future Shape in Chemical Education, Chem. Ed. Res. Pract.: 5, 229-245 (2004). 